\title{
Infantile fibrosarcoma-like tumor driven by novel RBPMS-MET fusion consolidated with cabozantinib
}

\author{
Ajay Gupta, ${ }^{1}$ Jennifer A. Belsky, ${ }^{1}$ Kathleen M. Schieffer ${ }^{2}{ }^{2}$ Kristen Leraas ${ }^{2}$ \\ Elizabeth Varga, ${ }^{2}$ Sean D. McGrath, ${ }^{2}$ Selene C. Koo, ${ }^{3,4}$ Vincent Magrini, ${ }^{2,5}$ \\ Richard K. Wilson, ${ }^{2,5}$ Peter White, ${ }^{2,5}$ Elaine R. Mardis, ${ }^{2,5}$ Kris R. Jatana, ${ }^{6,7}$ \\ Catherine E. Cottrell, ${ }^{2,4,5}$ and Bhuvana A. Setty ${ }^{1,5}$
}

\begin{abstract}
${ }^{1}$ Division of Hematology, Oncology, Blood and Marrow Transplant, ${ }^{2}$ The Steve and Cindy Rasmussen Institute for Genomic Medicine, ${ }^{3}$ Department of Pathology, Nationwide Children's Hospital, Columbus, Ohio 43205, USA; ${ }^{4}$ Department of Pathology, ${ }^{5}$ Department of Pediatrics, The Ohio State University, Columbus, Ohio 43210, USA; ${ }^{6}$ Department of Otolaryngology, Nationwide Children's Hospital, Columbus, Ohio 43205, USA; ${ }^{7}$ Department of Otolaryngology-Head and Neck Surgery, The Ohio State University, Columbus, Ohio 43210, USA
\end{abstract}

Abstract Infantile fibrosarcoma (IFS) is nearly universally driven by gene fusions involving the NTRK family. ETV6-NTRK3 fusions account for 85\% of alterations; the remainder are attributed to NTRK-variant fusions. Rarely, other genomic aberrations have been described in association with tumors identified as IFS or IFS-like. We describe the utility of genomic characterization of an IFS-like tumor. We also describe the successful treatment combination of VAC (vincristine, actinomycin, cyclophosphamide) with tyrosine kinase inhibitor (TKI) maintenance in this entity. This patient presented at birth with a right facial mass, enlarging at $1 \mathrm{mo}$ to $4.9 \times 4.5 \times 6.3 \mathrm{~cm}$. Biopsy demonstrated hypercellular fascicles of spindle cells with patchy positivity for smooth muscle actin (SMA) and negativity for S100, desmin, myogenin, and MyoD1. Targeted RNA sequencing identified a novel RBPMS-MET fusion with confirmed absence of ETV6-NTRK3, and the patient was diagnosed with an IFS-like tumor. A positron emission tomography (PET) scan was negative for metastatic disease. VAC was given for a duration of 10 mo. Resection at 13 mo of age demonstrated positive margins. Cabozantinib, a MET-targeting TKI, was initiated. The patient tolerated cabozantinib well and has no evidence of disease at 24 mo of age. We describe a novel RBPMS-MET driver fusion in association with a locally aggressive IFS-like tumor. MET functions as an oncogene and, when associated with the RNA binding protein RBPMS, forms an in-frame fusion product that retains the MET kinase domain. This fusion is associated with aberrant cell signaling pathway expression and subsequent malignancy. We describe treatment with cabozantinib in a patient with an IFS-like neoplasm.

[Supplemental material is available for this article.]

\section{INTRODUCTION}

Infantile fibrosarcoma (IFS) is the most common nonrhabdomyosarcoma soft-tissue sarcoma in infants (Bourgeois et al. 2000). Histologically, IFS consists of densely packed fascicles of spindle cells with frequently high mitotic activity and a nonspecific immunohistochemical staining pattern (Bourgeois et al. 2000). IFS is driven by gene fusions involving the NTRK (neurotrophic receptor tyrosine kinase) gene family and demonstrates favorable response 
COLD SPRING HARBOR Molecular Case Studies
Infantile fibrosarcoma driven by RBPMS-MET fusion to Trk (tropomyosin receptor kinase) inhibition (Albert et al. 2019). ETV6-NTRK3 fusions $(t(12 ; 15)(p 13 ; q 25))$ account for $\sim 85 \%$ of alterations, with the remainder attributed to NTRK-variant fusions (Albert et al. 2019).

IFS is usually localized, although metastases are more prevalent in patients with variant fusions, and overall prognosis is excellent for resectable tumors (Albert et al. 2019). In patients with unresectable disease, neoadjuvant therapy is standard. Prior to the era of highly selective Trk inhibitors, a rhabdomyosarcoma-like protocol was used. More recently, three patients with refractory IFS associated with LMNA-NTRK1 fusions were successfully treated with the oral MET/ALK/ROS1 tyrosine kinase inhibitor (TKI) crizotinib (Mody et al. 2015; Wong et al. 2016; Bender et al. 2019), buoyed by in vitro data of its action against TrkA (Vaishnavi et al. 2013). Additionally, an infant with ETV6-NTRK3 fusion-positive IFS responded to pazopanib suspension administered preoperatively (Yanagisawa et al. 2016).

Rarely, other genomic aberrations have been described in association with tumors identified as IFS. Based on the World Health Organization (WHO) 2013 definition of infantile fibrosarcoma, any histologically identical entity without a characteristic NTRK fusion could be considered IFS-like (Fletcher et al. 2013). Prior descriptions of IFS-like tumors revealed novel rearrangements involving the transmembrane receptor tyrosine kinase RET, including CLIP2RET, SPECC1L-RET, MYH10-RET, and KHDRBS1-RET (Church et al. 2018; Antonescu et al. 2019; Davis et al. 2020). Furthermore, four patients between 0 and 3 yr old with IFS-like tumors were identified to have BRAF rearrangements, including a CUX1-BRAF fusion (Kao et al. 2018). Given the breadth of described alterations in IFS and IFS-like tumors, next-generation sequencing methodologies are well-suited for detection and identification of appropriate targeted therapies (Kao et al. 2018).

\section{RESULTS}

\section{Clinical Presentation}

Our patient was born with a large right facial mass measuring $4.0 \times 4.1 \times 5.4 \mathrm{~cm}$ on magnetic resonance imaging (MRI) (Fig. 1A). A bedside fine-needle aspiration of the mass on the fourth day of life demonstrated spindled cells, which were smooth muscle actin (SMA)-positive and S100-negative. Because of the patient's age and tumor location, we opted for a short period of observation. At 1 month of age, repeat MRI showed enlargement of the mass to $4.9 \times 4.5 \times 6.3 \mathrm{~cm}$. Open biopsy demonstrated variably cellular fascicles of spindle cells; tumor cells were positive for SMA (patchy) and CD163, and negative for S100, desmin, CD34, myogenin, and MyoD1 (Fig. 2). Reverse transcription polymerase chain reaction (RT-PCR) for ETV6-NTRK3 was negative. Next-generation sequencing identified an RBPMS-MET fusion suspected to be pathogenic, and the patient was diagnosed with IFSlike disease. A positron emission tomography (PET) scan was negative for metastatic disease. Because of continued tumor growth, neoadjuvant chemotherapy was started at 7 wk of age with vincristine, actinomycin D, and cyclophosphamide (VAC). The patient's course was complicated by pneumatosis intestinalis, which resolved after a brief $50 \%$ dose reduction of vincristine and cyclophosphamide. She completed 10 mo of chemotherapy, at which point tumor burden decreased to $1.1 \mathrm{~cm}$ in its longest dimension in the right masticator space (Fig. 1B). At 13 mo of age, the residual mass was surgically resected en bloc, including a portion of the cheek skin and buccal mucosa, with meticulous dissection and preservation of the upper division of the facial nerve to maintain eye closure function. Final pathology demonstrated positive microscopic margins with no evidence of tumor necrosis. Postoperative imaging demonstrated postsurgical changes (Fig. 1C). Because of both the morbidity of additional surgery or radiation and the availability of tumor-specific genomic results, the decision was made to start cabozantinib, a MET-targeting TKI with liquid formulation, at $40 \mathrm{mg} / \mathrm{m}^{2} / \mathrm{d}$. 

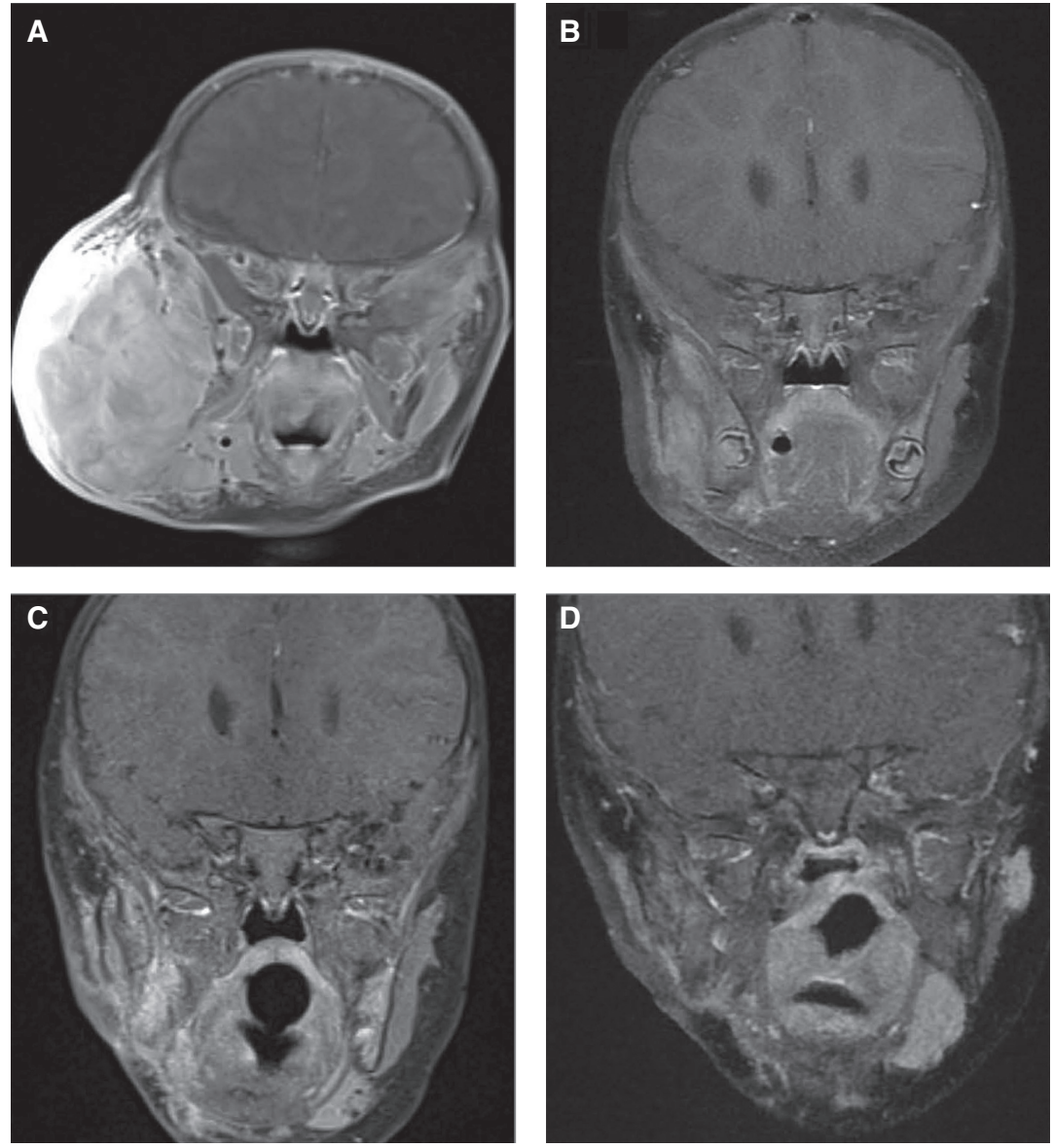

Figure 1. Tumor imaging. (A) Magnetic resonance imaging (MRI) at birth demonstrated a large right facial mass with a solid component measuring $4 \times 4.1 \times 5.4 \mathrm{~cm}$. The superior extent of the mass was over the right lateral frontal bone and temporal fossa, inferiorly below the inferior cortical margin of the right mandible, posteriorly to the external auditory canal, anteriorly into the nasolabial fold, without orbital or intracranial extension. (B) At $1 \mathrm{yr}$ of age and after vincristine, actinomycin D, and cyclophosphamide (VAC) chemotherapy, the mass decreased to $1.1 \mathrm{~cm}$ in its longest dimension in the right masticator space. This infiltrated and mildly expanded the masseter muscle with patchy tumefactive enhancement. There was also mild mass effect on the right parotid gland superolaterally and submandibular gland inferomedially with long-standing bone remodeling of the right zygomatic arch and mandibular neck/ramus. (C) At 13 mo of age and after surgical resection, imaging demonstrated extensive postoperative changes. Mild expansion of the right zygomatic arch and absence of the right submandibular gland was noted. The right parotid gland appeared smaller than the left. Stranding and contrast enhancement of the right preauricular soft tissues was noted with loss of subcutaneous fat. Mild heterogeneous enhancement surrounded the right zygomatic arch and right mandibular ramus. (D) At 20 mo of age after 5 mo of cabozantinib, imaging demonstrated stable postsurgical changes in the right face with no discrete mass lesion visualized.

Treatment has been tolerated well for 9 mo thus far without side effects, and our patient continues with no clinical or radiographic evidence of disease at 24 mo of age (Fig. 1D).

\section{Genomic Analyses}

Following consent on an IRB-approved protocol, the patient underwent comprehensive molecular profiling consisting of paired tumor/normal exome analysis and RNA sequencing. This included evaluation of germline and somatic single-nucleotide variation (SNV), small 

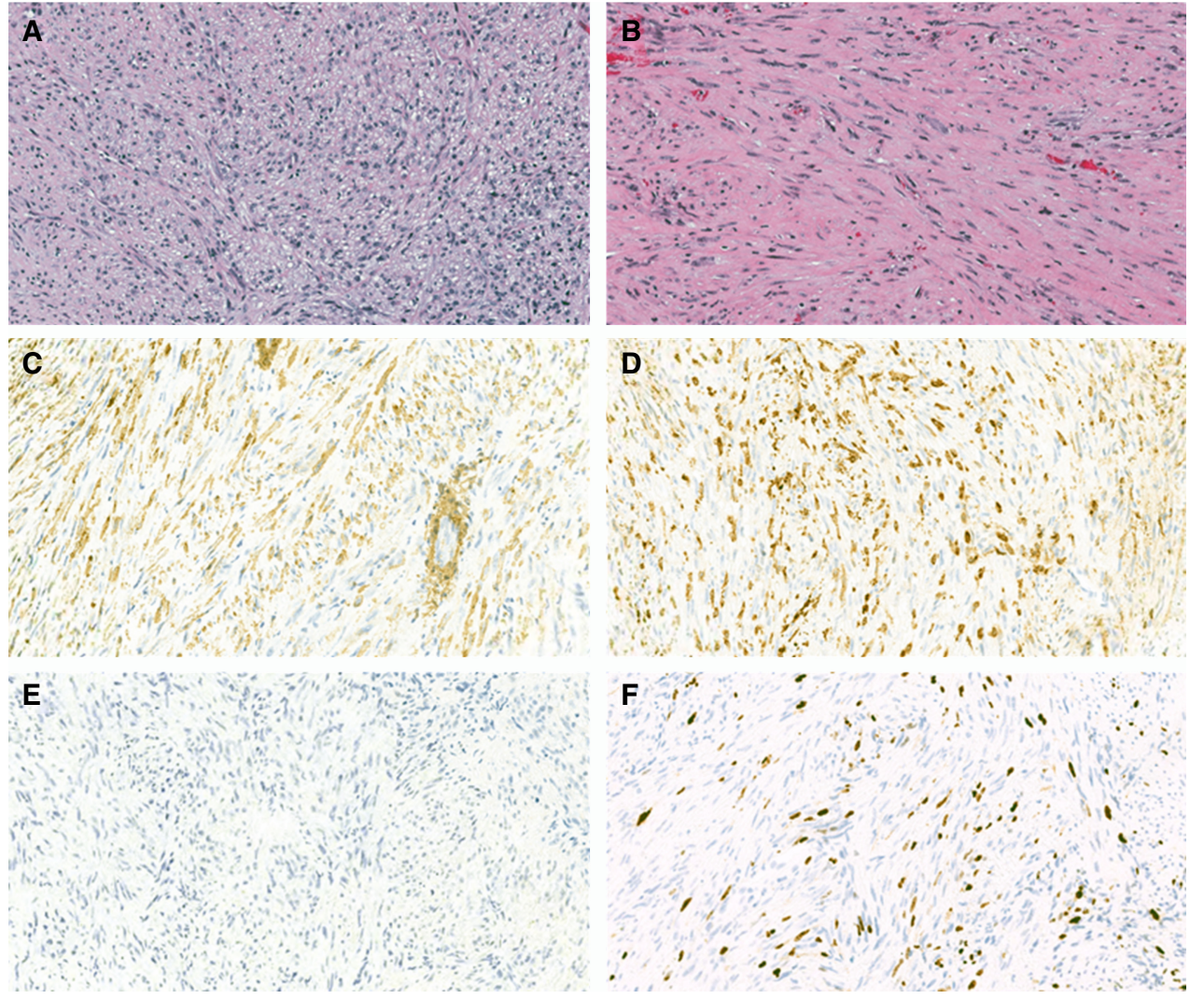

Figure 2. Histologic findings. The tumor is variably cellular, with $(A)$ areas containing cellular fascicles of ovoid to spindled cells with vacuolated nuclei and (B) paucicellular areas consisting of cells with long tapered nuclei within fibrous stroma. Mild-to-moderate nuclear pleomorphism and hyperchromasia are present. Thin-walled variably ectatic blood vessels are seen throughout. Tumor cells are positive on immunohistochemical stains for smooth muscle actin (C) and CD163 (D) and negative for desmin (E), S100, CD34, myogenin, MyoD1, and nuclear $\beta$-catenin (not shown). Ki-67 proliferation index is focally up to $20 \%(F)$.

indels, copy-number (CN) alterations, gene fusions, and aberrant gene expression. No pathogenic or likely pathogenic germline SNV or CN alterations were identified. Although no clearly medically meaningful somatic SNVs were identified (Supplemental Table 1), CN gain of Chromosomes 6, 8, 10, 11, 17, and 20 was found (Fig. 3A). Nonrandom gain among some of these chromosomes, in particular trisomy 11, is a cytogenetic abnormality commonly reported in IFS (Mandahl et al. 1989; Schofield et al. 1994). We identified the RBPMS-MET fusion through RNA sequencing with confirmation by RT-PCR and subsequent Sanger sequencing to establish reproducibility and verify the inferred translational protein frame (Fig. 3B). Isoform structure was determined by long read sequencing of the tumor. The inframe fusion event connected exon 5 of RBPMS (NM_006867) and exon 15 of MET (NM_000245) resulting in a 513-amino acid chimeric protein (Table 1; Fig. 3C). The fusion product resulted in juxtaposition of RBPMS, including the dimerization domain localized within the RNA recognition motif (RRM), to the intact MET protein kinase domain, a configuration predicted to result in kinase fusion gene activation (Stransky et al. 2014). IsoSeq long read sequencing of the RNA identified a second smaller fusion transcript (409 amino acids in length; Fig. 3C). The shorter transcript begins with RBPMS intron 4 sequence and encompasses the coding region of exon 5, with an identical breakpoint in MET to that 


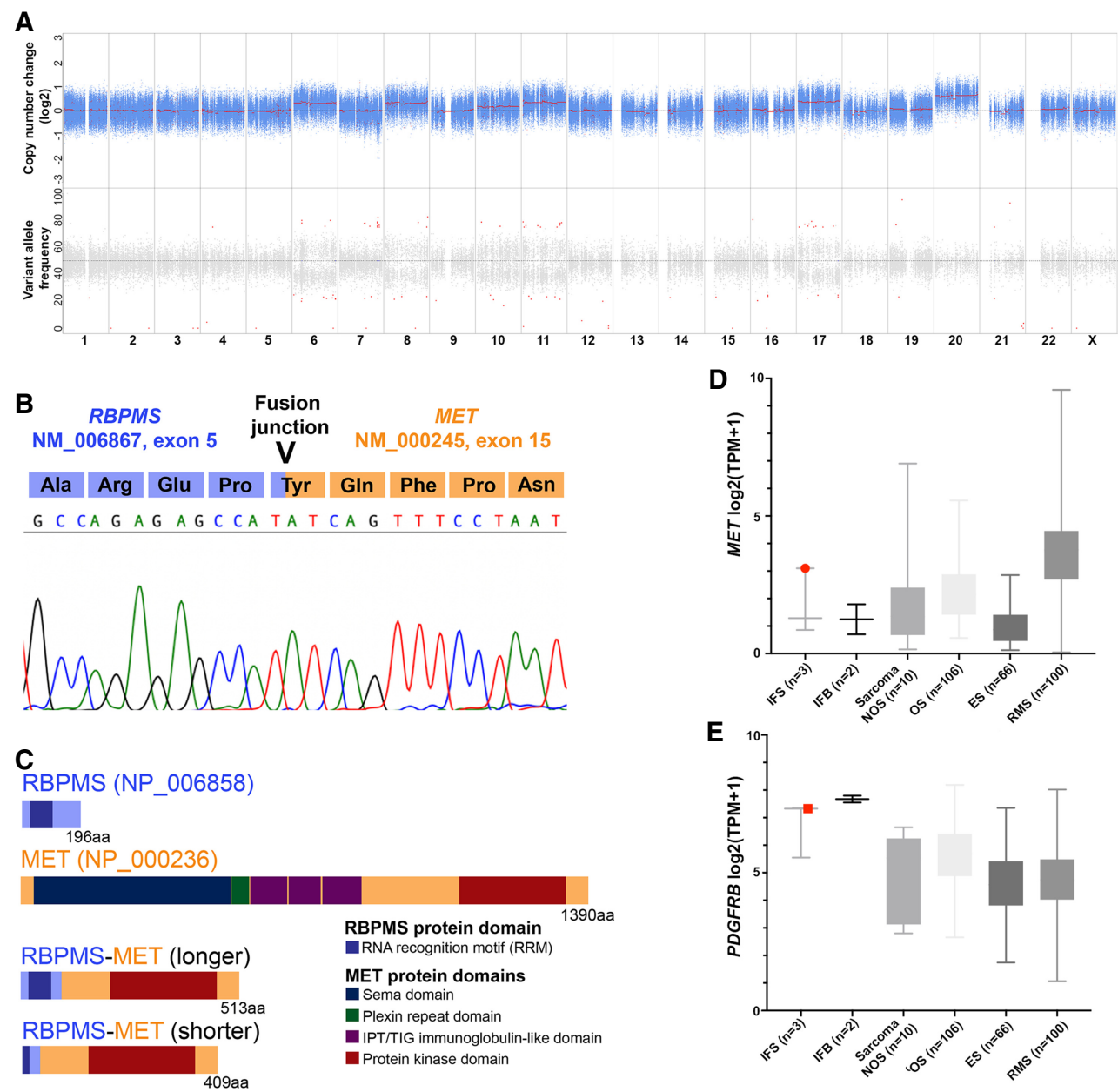

Figure 3. Molecular characterization of the primary tumor. (A) Somatic copy-number (CN) alterations. The top plot displays tumor $\mathrm{CN}$ relative to comparator normal (peripheral blood) in $\log _{2}$ scale. Blue points represent $\log _{2}$ values based on sequence depth in 100-bp windows. Red lines indicate segmented CN calls. The bottom plot displays tumor variant allele frequency for variants that are heterozygous in the normal, whereby points in red indicate significant loss of heterozygosity (LOH). The $x$-axis denotes the chromosome number. (B) Sanger sequencing chromatogram of the in-frame RBPMS-MET gene fusion. The corresponding amino acid sequence is described above the chromatogram. (C) Protein structure of the RBPMS-MET fusion. Gene expression of (D) MET and (E) PDGFRB among pediatric and adolescent/young adult soft-tissue tumors. The described patient case is shown as a red box. (IFS) Infantile fibrosarcoma, (IFB) infantile fibromatosis, (NOS) not otherwise specified, (OS) osteosarcoma, (ES) Ewing sarcoma, (RMS) rhabdomyosarcoma.

observed in the longer isoform. Analysis of gene expression data corresponded to aberrant signal transduction associated with the RBPMS-MET fusion. With the exception of rhabdomyosarcoma, MET gene expression was elevated relative to most of the soft-tissue tumors evaluated within a pediatric cohort comprised of institutional cases $(n=17)$ and those from the UCSC Treehouse Childhood Cancer Initiative $(n=270)$ (Fig. 3D), although this did not reach statistical significance $(P=0.68)$. We interpret elevated MET expression to be associated with the presence of the gene fusion, as the overexpression was skewed only toward those exons distal to the fusion breakpoint specifically exons 15-21 (Supplemental Fig. 1). Notably, the tumor demonstrated overexpression of PDGFRB ( $\log _{2}$ 


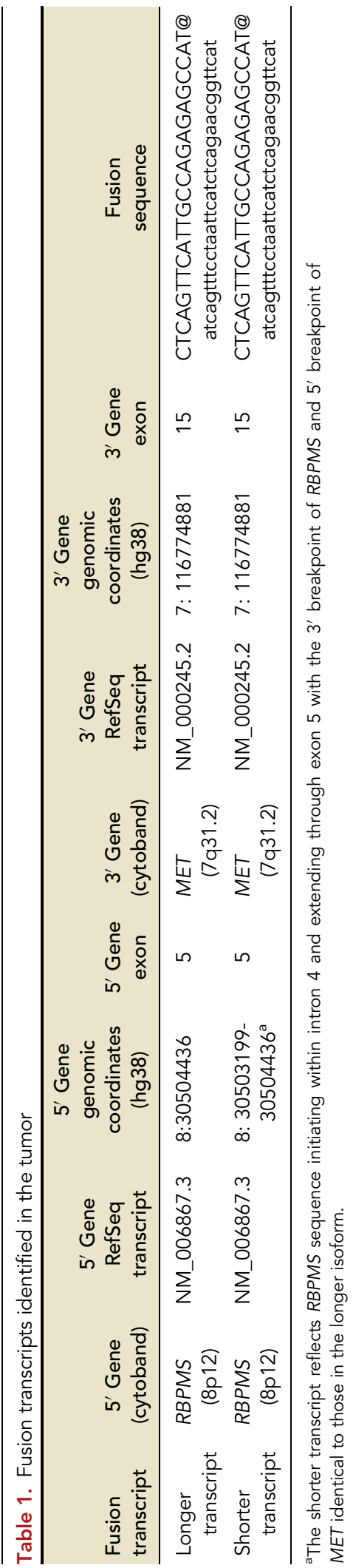


fold change: $3.40, P=0.003$ ), which was comparable to two infantile myofibromatosis cases with activating alterations in PDGFRB (Fig. 3E).

\section{DISCUSSION}

Herein, we report a unique case of an aggressive IFS-like tumor harboring a non-NTRK-variant fusion, RBPMS-MET, and the common aneuploidy changes associated with IFS, including trisomies 8, 11, 17, and 20. An RBPMS-MET fusion has only been described in a single adult with cholangiocarcinoma with similar breakpoints as described in our tumor (The ICGC/TCGA Pan-Cancer Analysis of Whole Genomes Consortium 2020). RNA-binding protein with multiple splicing (RBPMS) has been found to regulate cancer cell proliferation and migration (Fu et al. 2015) as well as smooth muscle plasticity (Sagnol et al. 2014). Under normal physiological conditions, the hepatocyte growth factor (HGF) ligand binds MET receptor and induces dimerization and autophosphorylation of the protein kinase domain, resulting in receptor signaling (Zhang et al. 2018). This activation induces cell proliferation, survival, growth, angiogenesis, and metastasis (Cui et al. 2011). In many receptor tyrosine kinase fusions, the $5^{\prime}$ gene partner contributes an oligomerization domain, such as a coiled-coil motif, which promote constitutively active and ligand-independent receptor activation (Du and Lovly 2018). The RRM domain of RBPMS is involved in both RNA recognition and homodimerization (Sagnol et al. 2014; Teplova et al. 2016). The in-frame fusion product retains the RRM and dimerization motifs of RBPMS and the protein kinase domain of MET, which is predicted to promote aberrant downstream signaling through Ras-MAPK and PI3K/AKT pathways. Activation of MET and downstream signaling through the PI3K/AKT and Ras-MAPK pathways occurs through tyrosine phosphorylation. Additionally, activation of other receptor tyrosine kinases, including PDGFRB, has been reported because of cross-talk of these receptors through trans-phosphorylation (Yeh et al. 2011; Lai et al. 2018). Using long read sequencing technologies, we also identified a shorter fusion isoform with a truncated RRM. The impact of this fusion on ligand-independent MET receptor activation is unknown, although given the likely impaired dimerization of RBPMS, this product may be hypothesized to have a lesser to noncontributory impact on downstream signaling and oncogenesis.

Genomic characterization and molecular diagnostics are expanding knowledge and treatment of IFS and IFS-like tumors. Classically, IFS is cytogenetically characterized by a translocation of Chromosomes 12p13 and 15q25, corresponding to an ETV6-NTRK3 gene fusion, and trisomy 11 (Sandberg and Bridge 2002), with occasional trisomies of 8, 17, and 20 (Sandberg and Bridge 2002). As mentioned previously, histologically identical entities without the classic gene fusion can be considered IFS-like and feature an assortment of novel rearrangements, now including an RBPMS-MET fusion with this report. Although sarcomas demonstrate the highest frequency of kinase fusions across 20 distinct solid tumor histologies (Stransky et al. 2014), MET fusions are infrequently reported as drivers. There is a single report of a TFG-MET fusion in an unusual S100-positive infantile spindle cell sarcoma (Flucke et al. 2017). In terms of RBPMS involvement, there are two prior reports of RBPMSNTRK3 fusion positive sarcomas: an adult with a fibrosarcoma-like uterine sarcoma (Chiang et al. 2018) and a 20-mo-old with a fibroblastic central nervous system (CNS) tumor (Torre et al. 2018). Additionally, RBPMS-NRG1 fusions have been described in adult lung adenocarcinoma and renal cell carcinoma (Jonna et al. 2019).

There is substantial literature over the past 35 years to support the use of neoadjuvant chemotherapy during the infancy period for IFS (Grier et al. 1985; Ninane et al. 1991). Such regimens have included VAC, as in our patient, with variable use of doxorubicin, ifosfamide, and etoposide (Kynaston et al. 1993; Russell et al. 2009). However, the use of TKls to treat sarcomas of infancy is rare. Imatinib has accrued the most experience, with 
documented use in a mast cell sarcoma in an 11-mo-old (Bautista-Quach et al. 2013) and dermatofibrosarcoma protuberans in 10- and 12-mo-old infants (Gooskens et al. 2010; Suzuki et al. 2011). Cabozantinib has been studied in COG phase I and II trials (ADVL1211 and ADVL1622) for children with recurrent or refractory solid tumors (Chuk et al. 2018), in which the youngest reported patient was 4 years old. Tolerability with extended disease control in children with neuroblastoma has been reported (Perisa et al. 2020). Although there were dose-limiting toxicities in the phase I trial at every dose level (including palmar-plantar erythrodysesthesia syndrome and mucositis), the toxicity profile overall resembled that of adults: diarrhea, fatigue, hypothyroidism, hypertension, weight loss, anorexia, and nausea (Chuk et al. 2018). Lab abnormalities included elevations in alanine aminotransferase, lipase, bilirubin, and proteinuria. Thus far, our patient has tolerated cabozantinib without significant clinical or laboratory toxicities.

In this patient's tumor, this fusion was associated with aberrant cell signaling pathway expression. Based on the biological mechanism associated with MET fusion, we are successfully treating our patient with the tyrosine kinase inhibitor cabozantinib. Patient-centric studies can improve our understanding of the mechanisms of disease and describe novel variants, the knowledge of which can benefit both the individual, as well as the larger patient population.

\section{METHODS}

\section{Patient Enrollment}

The infant was enrolled as part of an Institutional Review Board (IRB)-approved study at the Institute for Genomic Medicine at Nationwide Children's Hospital (NCH). Informed consent for comprehensive molecular analysis was provided by the parents. Peripheral blood (PB) was collected by routine venipuncture for genomic DNA extraction. Snap-frozen tumor tissue from the initial surgery was obtained for tumor DNA and RNA extraction.

\section{DNA Sequencing}

Enhanced exome sequencing was performed on DNA extracted from peripheral blood (comparator sample) and disease-involved tissue (snap-frozen tumor). Pathology estimated the sequenced section at 95\% tumor cellularity. Libraries were prepared using $100 \mathrm{ng}$ of input DNA beginning with enzymatic fragmentation, followed by end repair, 5' phosphorylation, A-tailing, and sequencing adapter ligation using NEB Ultra II FS (peripheral blood) and NEB Ultra II FS (snap-frozen) reagents (New England Biolabs). Target enrichment by hybrid capture was performed with IDT xGen Exome Research Panel v1.0 enhanced with the xGenCNV Backbone and Cancer-Enriched Panels-Tech Access (Integrated DNA Technologies). Paired-end 151-bp reads were generated on the Illumina HiSeq 4000. Secondary analysis was performed using Churchill, a comprehensive workflow for analysis of raw reads from genome alignment through to germline and somatic variant identification (Kelly et al. 2015). Reads were aligned to the human genome reference sequence (build GRCh37) using BWA (v0.7.15). Sequence alignments were refined according to community-accepted guidelines for best practices (https://gatk.broadinstitute.org/hc/en-us). Duplicate sequence reads were removed using samblaster-v.0.1.22, and local realignment was performed on the aligned sequence data using the Genome Analysis Toolkit (v3.7-0). Churchill's own deterministic implementation of base quality score recalibration was used. Germline variants were called using GATK's HaplotypeCaller. Average sequencing coverage depth for the tumor sample was $240 \times$ and $265 \times$ for the comparator peripheral blood sample (Supplemental Table 2). Somatic SNV and indel detection was performed using MuTect-2 
COLD SPRING HARBOR Molecular Case Studies
Infantile fibrosarcoma driven by RBPMS-MET fusion
(Cibulskis et al. 2013). Somatic variants in cancer-associated genes were identified (Zhang et al. 2015). Copy-number variation (CNV) was assessed using VarScan2 (Koboldt et al. 2012).

\section{RNA Sequencing}

In parallel, 500 ng of snap-frozen tumor RNA was subjected to DNase treatment and ribodepletion prior to using Illumina's TruSeq Stranded Total RNA Sample prep (performed with 2-min chemical fragmentation). An independent RNA-seq library was constructed for whole-transcriptome sequencing. Paired-end 151-bp reads were generated on the Illumina HiSeq 4000, and reads were aligned to the human genome reference sequence (GRCh38) with the resultant output representing 278,462,016 uniquely mapped reads (Supplemental Table 2). RNA sequence data were processed using an ensemble approach of seven fusion callers (STAR-Fusion [v.1.2.0] [Haas et al. 2019], MapSplice [v.2.2.1] [Wang et al. 2010], TopHat-Fusion [v.2.1.0] [Kim and Salzberg 2011], FusionCatcher [v.0.99.7c] [Nicorici et al. 2014], FusionMap [v.mono-2.10.9] [Ge et al. 2011], JAFFA [v.1.09] [Davidson et al. 2015], and SOAPfuse [v.1.27] [Jia et al. 2013]). Fusions were assessed for biological significance if identified by at least three tools and the fusion was rare ( $<10 \%$ frequency) within our internal cancer cohort. Fusion of genes located within $200 \mathrm{~kb}$ on the same chromosome strand were considered readthrough events and not evaluated further. A comprehensive literature review of the fusion genes was performed to determine the putative mechanism (i.e., activation due to fusion of an oncogene or loss of function of a tumor suppressor) and to identify previous reports of similar fusion events. Transcripts per million (TPM) values were generated from paired-end RNA sequence data using Salmon with bootstrapping set to 100 (Patro et al. 2017). Gene expression data were compared to publicly available RNA sequence data from the University of California Santa Cruz (UCSC) Treehouse Childhood Cancer Initiative (https ://treehousegenomics.soe.ucsc.edu/). Gene coverage was evaluated at the exon level. Briefly, a BED file was generated of exon coordinates using UCSC Genome Browser. Coverage was obtained from sorted BAM files using BEDtools (v.2.29.0) multiBamCov (Quinlan and Hall 2010). GenVisR was used to visualize coverage depth across RBPMS and MET (Skidmore et al. 2016).

\section{Sanger Sequencing of RBPMS-MET Fusion}

We used 500 ng of RNA with MultiScribe reverse transcriptase (ThermoFisher) and random hexamers (Applied Biosystems) for RT-PCR. PCR of cDNA was performed with the forward primer in RBPMS exon 5 (5'ATCCGCTTCGATCCTGAAAT 3') and reverse primer in MET exon 15 (5'TGACTTCATTGAAATGCACAA 3'). PCR product was purified using the QIAquick purification kit (QIAGEN). Forward and reverse Sanger sequencing reactions were performed with the Big Dye v3.1 terminator mix (ThermoFisher). Sequencing was performed on an Applied Biosystems 3130 instrument.

\section{SMARTer cDNA Synthesis}

First-strand cDNA was prepared from $1 \mu \mathrm{g}$ tumor total RNA in a single SMARTer (SMARTer PCR cDNA Synthesis Kit, Takara Bio USA) according to the company protocol. First-strand cDNA was diluted with $90 \mu \mathrm{L}$ Buffer EB (QIAGEN). Second-strand synthesis was performed in a large-scale PCR reaction with a universal PCR primer added during first-strand cDNA synthesis. Second-strand synthesis reactions were prepared using PrimeSTAR GXL DNA polymerase in the following reaction: $10 \mu \mathrm{L}$ diluted first-strand cDNA, $0.2 \mathrm{mM}$ dNTPs, $0.24 \mu \mathrm{M}$ 5' PCR Primer IIA, 1.25 units PrimeSTAR GXL DNA Polymerase in 1× PrimeSTAR GXL Buffer with PCR cycling conditions of $30 \mathrm{sec}$ at $98^{\circ} \mathrm{C}, 12 \times\left(10 \mathrm{sec}\right.$ at $98^{\circ} \mathrm{C}, 15 \mathrm{sec}$ at $65^{\circ} \mathrm{C}$, $10 \mathrm{~min}$ at $68^{\circ} \mathrm{C}$ ) and $5 \mathrm{~min}$ at $68^{\circ} \mathrm{C}$. Large-scale PCR reactions were combined after 
COLD SPRING HARBOR Molecular Case Studies
Infantile fibrosarcoma driven by RBPMS-MET fusion amplification and concentrated with $1 \times$ Pacific Biosciences AMPure. Concentrated secondstrand cDNA was eluted in $50 \mu \mathrm{L}$ Buffer EB. The concentrated second-strand cDNA concentration was determined with Qubit $1 \times$ dsDNA HS Working Solution kit and the Qubit 3.0 fluorometer (Thermo Fisher Scientific Inc.).

\section{cDNA Size Fractionation}

Concentrated second-strand cDNA was size-fractionated with a double-sided SPRIselect reaction. Initially, $50 \mu \mathrm{L}$ second-strand cDNA was combined with $20 \mu \mathrm{L}$ SPRlselect reagent $(0.4 \times)$. The supernatant of this reaction was transferred to a clean tube and combined with $30 \mu \mathrm{L}$ SPRIselect $(0.4 / 1 \times)$. This sequential reaction results in cDNA sized at $>2 \mathrm{~kb}$ and $0.2-2 \mathrm{~kb}$, respectively. The size-fractionated cDNA concentration was determined with Qubit $1 \times$ dsDNA HS Working Solution kit and the Qubit 3.0 fluorometer and the average library size for the cDNA size fractions was determined with the Agilent High Sensitivity DNA Kit on the Bioanalyzer 2100.

\section{SMRTbell Template Prep}

Molar concentrations for each cDNA fraction were calculated using the average cDNA size determined by the 2100 Bioanalyzer and the mass as determined by Qubit. Prior to PacBio library preparation, an equal molar dilution was prepared from the $0.4 \times$ SPRlselect and 0.4/1 × SPRIselect fractions. A SMRTbell Template Prep Kit 1.0 library was prepared from $1 \mathrm{\mu g}$ size-fractionated cDNA according to the PacBio Iso-Seq protocol (PN 101-070-200 Version 05 [November 2017]). The final library was diluted 1:5 with Buffer EB and assessed for DNA concentration by Qubit dsDNA HS and size by 2100 Bioanalyzer High Sensitivity DNA kit.

\section{Pacific Biosciences Sequel Iso-Seq Sequencing}

Library metrics metadata values including available volume, concentration, and average insert size were entered into the SMRT Link Sample Setup worksheet. The worksheet calculates the required sequencing primer $\mathrm{v} 3$ annealing and polymerase binding conditions for each SMRTbell library. The SMRTbell library was complexed with the Sequel Binding Kit 2.1, purified and concentrated according to the AMPure PB protocol loaded at 8 pM with the diffusion protocol. Sequencing was performed with two v2 LR SMRT Cells, v2.1 Sequencing Reagents, and 4-h preextension followed by $20-\mathrm{h}$ movie collection. Coverage metrics are provided in Supplemental Table 2.

\section{Iso-Seq Analysis in SMRT Link}

Both SMRT Cells were analyzed with the Iso-Seq application in SMRT Link version 8.0.0.79519 with the default parameters (Maximum CCS Read Length =15,000, Minimum CCS Read Length $=50$, Polish CCS $=$ false, Filters to add to the DataSet = none, Minimum Accuracy for High-Quality Isoforms $=0.99$, Maximum Fuzzy Junction Difference $(b p)=5$, Ignore BioSamples records from Run Design = false, Minimum Mapped Concordance $(\%)=95$, Minimum Mapped Coverage $(\%)=99$, Minimum Mapped Length (bp) $=50$, Require and Trim Poly(A) Tail=true, Run Clustering=true). The resulting BAM file of Mapped High-Quality Isoforms was visualized in the Integrated Genomics Viewer version 2.8.0 to identify RBPMS-MET fusion transcripts. 
Competing Interest Statement The authors have declared no competing interest.

Received June 8, 2020; accepted in revised form July 17, 2020.

\section{ADDITIONAL INFORMATION}

\section{Data Deposition and Access}

Molecular data from the diagnostic case have been deposited to dbGAP accession phs001820.v1.p1., under submitter: Institute for Genomic Medicine (IGM) Clinical Laboratory, Nationwide Children's Hospital. Details are provided in the Methods. The RBPMS-MET fusion has been submitted to ClinVar (https://www.ncbi.nlm.nih.gov/clinvar/) under accession number SCV001433870.

\section{Ethics Statement}

All patients or their guardians provided written informed consent for genomic sequencing. This research is under a protocol approved by the Institutional Review Board at Nationwide Children's Hospital (IRB17-00206).

\section{Acknowledgments}

This research was supported by the Diagnostic Immunology Laboratory, Nationwide Children's Hospital. We thank the Nationwide Foundation Pediatric Innovation Fund for generously supporting sequencing, data production, and analysis. We would like to acknowledge the Treehouse Childhood Cancer Initiative at the University of California Santa Cruz Genomics Institute.

\section{Author Contributions}

A.G. wrote the first manuscript. A.G., J.A.B., K.M.S., C.E.C., S.C.K., K.R.J., and B.A.S. wrote, reviewed, discussed, edited, and revised the manuscript. V.M., S.D.M., K.L., E.V., R.K.W., P. W., E.R.M., C.E.C., and K.M.S. performed data analysis and interpretation.

\section{Funding}

Funding was provided by the Nationwide Foundation Pediatric Innovation Fund.

\section{REFERENCES}

Albert CM, Davis JL, Federman N, Casanova M, Laetsch TW. 2019. TRK fusion cancers in children: a clinical review and recommendations for screening. J Clin Oncol 37: 513-524. doi:10.1200/JCO.18.00573

Antonescu CR, Dickson BC, Swanson D, Zhang L, Sung YS, Kao YC, Chang WC, Ran L, Pappo A, Bahrami A, et al. 2019. Spindle cell tumors with RET gene fusions exhibit a morphologic spectrum akin to tumors with NTRK gene fusions. Am J Surg Pathol 43: 1384-1391. doi:10.1097/PAS.0000000000001297

Bautista-Quach MA, Booth CL, Kheradpour A, Zuppan CW, Rowsell EH, Weiss L, Wang J. 2013. Mast cell sarcoma in an infant: a case report and review of the literature. J Pediatr Hematol/Oncol 35: 315-320. doi:10 .1097/MPH.0b013e318279e392

Bender J, Anderson B, Bloom DA, Rabah R, McDougall R, Vats P, Mody R. 2019. Refractory and metastatic infantile fibrosarcoma harboring LMNA-NTRK1 fusion shows complete and durable response to crizotinib. Cold Spring Harb Mol Case Stud 5: 1-10. doi:10.1101/mcs.a003376

Bourgeois JM, Knezevich SR, Mathers JA, Sorensen PHB. 2000. Molecular detection of the ETV6-NTRK3 gene fusion differentiates congenital fibrosarcoma from other childhood spindle cell tumors. Am J Surg Pathol 24: 937-946. doi:10.1097/00000478-200007000-00005

Chiang S, Cotzia P, Hyman DM, Drilon A, Tap WD, Zhang L, Hechtman JF, Frosina D, Jungbluth AA, Murali R, et al. 2018. NTRK fusions define a novel uterine sarcoma subtype with features of fibrosarcoma. Am J Surg Pathol 42: 791-798. doi:10.1097/PAS.0000000000001055

Chuk MK, Widemann BC, Minard CG, Liu X, Kim AR, Bernhardt MB, Kudgus RA, Reid JM, Voss SD, Blaney S, et al. 2018. A phase 1 study of cabozantinib in children and adolescents with recurrent or refractory solid tumors, including CNS tumors: trial ADVL1211, a report from the children's oncology group. Pediatr Blood Cancer 65: 1-7. doi:10.1002/pbc.27077 
Church AJ, Calicchio ML, Nardi V, Skalova A, Pinto A, Dillon DA, Gomez-Fernandez CR, Manoj N, Haimes JD, Stahl JA, et al. 2018. Recurrent EML4-NTRK3 fusions in infantile fibrosarcoma and congenital mesoblastic nephroma suggest a revised testing strategy. Mod Patho/ 31: 463-473. doi:10.1038/modpathol.2017.127

Cibulskis K, Lawrence MS, Carter SL, Sivachenko A, Jaffe D, Sougnez C, Gabriel S, Meyerson M, Lander ES, Getz G. 2013. Sensitive detection of somatic point mutations in impure and heterogeneous cancer samples. Nat Biotechnol 31: 213-219. doi:10.1038/nbt.2514

Cui JJ, Tran-Dubé M, Shen H, Nambu M, Kung PP, Pairish M, Jia L, Meng J, Funk L, Botrous I, et al. 2011. Structure based drug design of crizotinib (PF-02341066), a potent and selective dual inhibitor of mesenchymal-epithelial transition factor (c-MET) kinase and anaplastic lymphoma kinase (ALK). J Med Chem 54: 6342-6363. doi:10.1021/jm2007613

Davidson NM, Majewski IJ, Oshlack A. 2015. JAFFA: high sensitivity transcriptome-focused fusion gene detection. Genome Med 7: 43. doi:10.1186/s13073-015-0167-x

Davis JL, Vargas SO, Rudzinski ER, López Marti JM, Janeway K, Forrest S, Winsnes K, Pinto N, Yang SE, VanSandt M, et al. 2020. Recurrent RET gene fusions in paediatric spindle mesenchymal neoplasms. Histopathology 76: 1032-1041. doi:10.1111/his.14082

Du Z, Lovly CM. 2018. Mechanisms of receptor tyrosine kinase activation in cancer. Mol Cancer 17: 58. doi:10 .1186/s12943-018-0782-4

Fletcher CDM, Bridge JA, Hogendoorn P, Mertens FJ. 2013. WHO classification of tumours of soft tissue and bone (IARC WHO Classification of Tumours). World Health Organisation, Geneva.

Flucke U, van Noesel MM, Wijnen M, Zhang L, Chen CL, Sung YS, Antonescu CR. 2017. TFG-MET fusion in an infantile spindle cell sarcoma with neural features. Genes Chromosomes Cancer 56: 663-667. doi:10.1002/gcc.22470

Fu J, Cheng L, Wang Y, Yuan P, Xu X, Ding L, Zhang H, Jiang K, Song H, Chen Z, et al. 2015. The RNA-binding protein RBPMS1 represses AP-1 signaling and regulates breast cancer cell proliferation and migration. Biochim Biophys Acta 1853: 1-13. doi:10.1016/j.bbamcr.2014.09.022

Ge H, Liu K, Juan T, Fang F, Newman M, HoeckW. 2011. FusionMap: detecting fusion genes from next-generation sequencing data at base-pair resolution. Bioinformatics 27: 1922-1928. doi:10.1093/bioinformatics/btr310

Gooskens SLM, Oranje AP, van Adrichem LNA, de Waard-van der Spek FB, den Hollander JC, van de Ven CP, van den Heuvel-Eibrink MM. 2010. Imatinib mesylate for children with dermatofibrosarcoma protuberans (DFSP). Pediatr Blood Cancer 55: 369-373. doi:10.1002/pbc.22494

Grier HE, Perez-Atayde AR, Weinstein HJ. 1985. Chemotherapy for inoperable infantile fibrosarcoma. Cancer 56: 1507-1510. doi:10.1002/1097-0142(19851001)56:7\&lt;1507::AID-CNCR2820560705>3.0.CO;2-7

Haas BJ, Dobin A, Li B, Stransky N, Pochet N, Regev A. 2019. Accuracy assessment of fusion transcript detection via read-mapping and de novo fusion transcript assembly-based methods. Genome Biol 20: 213. doi:10.1186/s13059-019-1842-9

Jia W, Qiu K, He M, Song P, Zhou Q, Zhou F, Yu Y, Zhu D, Nickerson ML, Wan S, et al. 2013. SOAPfuse: an algorithm for identifying fusion transcripts from paired-end RNA-seq data. Genome Biol 14: R12. doi:10 .1186/gb-2013-14-2-r12

Jonna S, Feldman RA, Swensen J, Gatalica Z, Korn WM, Borghaei H, Ma PC, Nieva JJ, Spira Al, Vanderwalde AM, et al. 2019. Detection of NRG1 gene fusions in solid tumors. Clin Cancer Res 25: 4966-4972. doi:10 1158/1078-0432.CCR-19-0160

Kao YC, Fletcher CDM, Alaggio R, Wexler L, Zhang L, Sung YS, Orhan D, Chang W-C, Swanson D, Dickson BC, et al. 2018. Recurrent BRAF gene fusions in a subset of pediatric spindle cell sarcomas-expanding the genetic spectrum of tumors with overlapping features with infantile fibrosarcoma. Am J Surg Pathol 42: 28-38. doi:10.1097/PAS.0000000000000938

Kelly BJ, Fitch JR, Hu Y, Corsmeier DJ, Zhong H, Wetzel AN, Nordquist RD, Newsom DL, White P. 2015. Churchill: an ultra-fast, deterministic, highly scalable and balanced parallelization strategy for the discovery of human genetic variation in clinical and population-scale genomics. Genome Biol 16: 6. doi:10.1186/ s13059-014-0577-x

Kim D, Salzberg SL. 2011. TopHat-fusion: an algorithm for discovery of novel fusion transcripts. Genome Biol 12: R72. doi:10.1186/gb-2011-12-8-r72

Koboldt DC, Zhang Q, Larson DE, Shen D, McLellan MD, Lin L, Miller CA, Mardis ER, Ding L, Wilson RK. 2012. VarScan 2: somatic mutation and copy number alteration discovery in cancer by exome sequencing. Genome Res 22: 568-576. doi:10.1101/gr.129684.111

Kynaston JA, Malcolm AJ, Craft AW, Davies SM, Morris Jones PH, King DJ, Mitchell CD, Oakhill A, Stiller CA. 1993. Chemotherapy in the management of infantile fibrosarcoma. Med Pediatr Oncol 21: 488-493. doi:10.1002/mpo.2950210706

Lai Y, Zhao Z, Zeng T, Liang X, Chen D, Duan X, Zeng G, Wu W. 2018. Crosstalk between VEGFR and Cabozantinib for relapsed neuroblastoma: single institution case series other receptor tyrosine kinases for TKI therapy of metastatic renal cell carcinoma. Cancer Cell Int 18: 31. doi:10.1186/s12935-018-0530-2

Mandahl N, Heim S, Rydholm A, Willen H, Mitelman F. 1989. Nonrandom numerical chromosome aberrations (+8, $+11,+17,+20)$ in infantile fibrosarcoma. Cancer Genet Cytogenet doi:10.1016/0165-4608(89)90156-8 
Mody RJ, Wu YM, Lonigro RJ, Cao X, Roychowdhury S, Vats P, Frank KM, Prensner JR, Asangani I, Palanisamy $\mathrm{N}$, et al. 2015. Integrative clinical sequencing in the management of refractory or relapsed cancer in youth. J Am Med Assoc 314: 913-925. doi:10.1001/jama.2015.10080

Nicorici D, Şatalan M, Edgren H, Kangaspeska S, Murumägi A, Kallioniemi O, Virtanen S, Kilkku O. 2014. FusionCatcher-a tool for finding somatic fusion genes in paired-end RNA-sequencing data. BioRxiv doi:10.1101/011650

Ninane J, Rombouts JJ, Cornu G. 1991. Chemotherapy for infantile fibrosarcoma. Med Pediatr Oncol 19: 209. doi:10.1002/mpo.2950190313

Patro R, Duggal G, Love MI, Irizarry RA, Kingsford C. 2017. Salmon provides fast and bias-aware quantification of transcript expression. Nat Methods 14: 417-419. doi:10.1038/nmeth.4197

Perisa MP, Storey M, Streby KA, Ranalli MA, Skeens M, Shah N. 2020. Cabozantinib for relapsed neuroblastoma: single institution case series. Pediatr Blood Cancer 67: e28317. doi:10.1002/pbc.28317

Quinlan AR, Hall IM. 2010. BEDTools: a flexible suite of utilities for comparing genomic features. Bioinformatics 26: 841-842. doi:10.1093/bioinformatics/btq033

Russell H, John Hicks M, Bertuch AA, Chintagumpala M. 2009. Infantile fibrosarcoma: clinical and histologic responses to cytotoxic chemotherapy. Pediatr Blood Cancer 53: 23-27. doi:10.1002/pbc.21981

Sagnol S, Yang Y, Bessin Y, Allemand F, Hapkova I, Notarnicola C, Guichou JF, Faure S, Labesse G, De Santa Barbara P. 2014. Homodimerization of RBPMS2 through a new RRM-interaction motif is necessary to control smooth muscle plasticity. Nucleic Acids Res 42: 10173-10184. doi:10.1093/nar/gku692

Sandberg AA, Bridge JA. 2002. Updates on the cytogenetics and molecular genetics of bone and soft tissue tumors: congenital (infantile) fibrosarcoma and mesoblastic nephroma. Cancer Genet Cytogenet 132: 1 13. doi:10.1016/s0165-4608(01)00528-3

Schofield DE, Fletcher JA, Grier HE, Yunis EJ. 1994. Fibrosarcoma in infants and children. Application of new techniques. Am J Surg Pathol 18: 14-24. doi:10.1097/00000478-199401000-00002

Skidmore ZL, Wagner AH, Lesurf R, Campbell KM, Kunisaki J, Griffith OL, Griffith M. 2016. GenVisR: genomic visualizations in R. Bioinformatics 32: 3012-3014. doi:10.1093/bioinformatics/btw325

Stransky N, Cerami E, Schalm S, Kim JL, Lengauer C. 2014. The landscape of kinase fusions in cancer. Nat Commun 5: 4846. doi:10.1038/ncomms5846

Suzuki D, Kobayashi R, Yasuda K, Yamamoto H, Morioka K, Mikawa M, Kobayashi K. 2011. Congenital dermatofibrosarcoma protuberans in a newborn infant with a massive back tumor: favorable effects of oral imatinib on the control of residual tumor growth. J Pediatr Hematol/Oncol 33: 304-306. doi:10.1097/MPH $.0 \mathrm{~b} 013 \mathrm{e} 31822 \mathrm{~d} 4 \mathrm{~d} 21$

Teplova M, Farazi TA, Tuschl T, Patel DJ. 2016. Structural basis underlying CAC RNA recognition by the RRM domain of dimeric RNA-binding protein RBPMS. Q Rev Biophys 49: e1. doi:10.1017/S0033583515000207

The ICGC/TCGA Pan-Cancer Analysis of Whole Genomes Consortium. 2020. Pan-cancer analysis of whole genomes. Nature 578: 82-93. doi:10.1038/s41586-020-1969-6

Torre M, Jessop N, Hornick JL, Alexandrescu S. 2018. Expanding the spectrum of pediatric NTRK-rearranged fibroblastic tumors to the central nervous system: a case report with RBPMS-NTRK3 fusion. Neuropathology 38: 624-630. doi:10.1111/neup.12513

Vaishnavi A, Capelletti M, Le AT, Kako S, Butaney M, Ercan D, Mahale S, Davies KD, Aisner DL, Pilling AB, et al. 2013. Oncogenic and drug-sensitive NTRK1 rearrangements in lung cancer. Nat Med 19: 1469-1472. doi:10.1038/nm.3352

Wang K, Singh D, Zeng Z, Coleman SJ, Huang Y, Savich GL, He X, Mieczkowski P, Grimm SA, Perou CM, et al. 2010. MapSplice: accurate mapping of RNA-seq reads for splice junction discovery. Nucleic Acids Res 38: e178. doi:10.1093/nar/gkq622

Wong V, Pavlick D, Brennan T, Yelensky R, Crawford J, Ross JS, Miller VA, Malicki D, Stephens PJ, Ali SM, et al. 2016. Evaluation of a congenital infantile fibrosarcoma by comprehensive genomic profiling reveals an LMNA-NTRK1 gene fusion responsive to crizotinib. J Natl Cancer Inst 108: 10-12. doi:10.1093/jnci/djv307

Yanagisawa R, Noguchi M, Fujita K, Sakashita K, Sano K, Ogiso Y, Katsuyama Y, Kondo Y, Komori K, Fujihara I, et al. 2016. Preoperative treatment with pazopanib in a case of chemotherapy-resistant infantile fibrosarcoma. Pediatr Blood Cancer 63: 348-351. doi:10.1002/pbc.25733

Yeh C-Y, Shin S-M, Yeh H-H, Wu T-J, Shin J-W, Chang T-Y, Raghavaraju G, Lee C-T, Chiang J-H, Tseng VS, et al, 2011. Transcriptional activation of the Axl and PDGFR- $\alpha$ by c-met through a Ras- and Src-independent mechanism in human bladder cancer. BMC Cancer 11: 139. doi:10.1186/1471-2407-11-139.

Zhang J, Walsh MF, Wu G, Edmonson MN, Gruber TA, Easton J, Hedges D, Ma X, Zhou X, Yergeau DA, et al. 2015. Germline mutations in predisposition genes in pediatric cancer. N Engl J Med 373: 2336-2346. doi:10.1056/NEJMoa1508054

Zhang Y, Xia M, Jin K, Wang S, Wei H, Fan C, Wu Y, Li X, Li X, Li G, et al. 2018. Function of the c-Met receptor tyrosine kinase in carcinogenesis and associated therapeutic opportunities. Mol Cancer 17: 45. doi:10 $.1186 / \mathrm{s} 12943-018-0796-y$ 


\section{COLD SPRING HARBOR Molecular Case Studies}

\section{Infantile fibrosarcoma-like tumor driven by novel RBPMS-MET fusion consolidated with cabozantinib}

Ajay Gupta, Jennifer A. Belsky, Kathleen M. Schieffer, et al.

Cold Spring Harb Mol Case Stud 2020, 6: a005645

Access the most recent version at doi: $10.1101 / \mathrm{mcs} . \mathrm{a} 005645$
Supplementary http://molecularcasestudies.cshlp.org/content/suppl/2020/10/07/mcs.a005645.D Material C1

References This article cites 51 articles, 3 of which can be accessed free at: http://molecularcasestudies.cshlp.org/content/6/5/a005645.full.html\#ref-list-1

License This article is distributed under the terms of the Creative Commons Attribution-NonCommercial License, which permits reuse and redistribution, except for commercial purposes, provided that the original author and source are credited.

Email Alerting Receive free email alerts when new articles cite this article - sign up in the box at the Service top right corner of the article or click here. 